\title{
Кирюнин А.И. \\ К вопросу об оценке эффективности работы структурных подразделений по обслуживанию устройств инфраструктуры на основе методологии УРРАН
}

ФГБОУ ВО «Ростовский государственный университет путей сообщения» (Россия, Ростов-на-Дону)

doi: 10.18411/lj-04-2021-278

\section{Аннотация}

В данной статье проанализирована существующая методика оценки эффективности работы структурных подразделений по обслуживанию устройств инфраструктуры на основе методологии УРРАН, обоснована необходимость ее корректировки.

Ключевые слова: надежность, анализ рисков, управление ресурсами, показатели эффективности.

\section{Abstract}

This article analyzes the existing methodology for evaluating the effectiveness of structural units for servicing infrastructure devices based on the URRAN methodology, and justifies the need for its adjustment.

Keywords: reliability, hazard analysis, asset management, efficiency parameter.

Как известно качество перевозочного процесса на железнодорожном транспорте определяется быстротой и безопасностью доставки грузов и пассажиров к месту назначения. Оба эти показателя зависят от надежности функционирования систем обеспечения движения поездов (СОДП). Отказы данных систем приводят к задержке поездов, а в худшем случае, могут приводить к аварийным ситуациям и катастрофам.

Поэтому обеспечение надежности и безопасности движения поездов является одной из приоритетных задач ОАО «РЖД». Для решения данной задачи с 2010 г. в ОАО «РЖД» реализуется методология УРРАН - комплексное решение по управлению активами на основе управления ресурсами, рисками и надежностью на стадиях жизненного цикла объектов инфраструктуры и подвижного состава.

Одной из задач УРРАН является задача распределения имеющихся ресурсов на основе снижения рисков функционирования систем и устройств железнодорожной инфраструктуры в рамках заданных значений показателей надежности и безопасности. Оценка рисков в методологии УРРАН базируется на, оценке проектных, допустимых и фактических (достигнутых) показателей надежности и безопасности устройств железнодорожной инфраструктуры и последующем их сопоставлении между собой.

В настоящее время методология УРРАН используется не только для оценки степени надежности и безопасности устройств железнодорожной инфраструктуры, но и для оценки эффективности работы структурных подразделений по обслуживанию этих устройств [1].

В соответствии с предложенной методикой вся инфраструктура железнодорожного транспорта рассматривается как совокупность типовых объектов инфраструктуры (ТОИ), распределённых по территориальному (например, перегонные и станционные в пределах какого-либо участка) и функциональному (например, в хозяйстве автоматики и телемеханики - комплекс технических средств управления стрелкой на станции и блок-участок на перегоне) признакам.

Такой подход, по мнению авторов методики, позволяет оценить эффективность работы различных структурных подразделений ОАО «РЖД» в зависимости от качества функционирования ТОИ конкретного хозяйства.

В соответствии с методологией УРРАН [2] степень надежности и безопасности ТОИ, а, следовательно, и СОДП, может быть определена на основе анализа 
соотношения допустимых, проектных и фактических значений таких основных показателей надежности, как коэффициент простоя $K_{n}$, интенсивность потока отказов $\lambda$ (или среднего времени наработки до отказа $T_{o}$, т.к. $T_{o}=1 / \lambda$ ) и среднее время до восстановления работоспособности Тв.

Сравнение допустимого и проектного уровней выбранных показателей позволяет оценить, удовлетворяет ли надежность технических средств перегонов и станций требованиям перевозочного процесса или они подлежат модернизации.

Сопоставление проектного и фактического уровней этих показателей информирует о том, насколько эксплуатационные подразделения обеспечивают изначально заложенные в технических средствах показатели надежности при проведении регламентных и восстановительных работ.

Сравнение допустимого и фактического уровней дает возможность определить, соответствует ли последний из них требованиям перевозочного процесса.

Методология УРРАН предусматривает шесть возможных вариантов соотношения различных значений интенсивности потока отказов и соответственно шесть возможных сценариев принятия управленческих решений по содержанию устройств железнодорожной инфраструктуры [3].

Для объективного формирования инвестиционной программы модернизации технических средств и оценки эффективности деятельности эксплуатационных подразделений, по мнению авторов методики, простого сравнения трех значений выбранных показателей надежности недостаточно. Для выработки обоснованных решений необходима оценка влияния на коэффициент простоя показателей безотказности и ремонтопригодности в отдельности.

Достичь требуемого значения коэффициента простоя можно путем сокращения среднего времени до восстановления работоспособности Тв или снижения величины интенсивности потока отказов $\lambda$ за счет совершенствования процесса технического обслуживания устройств.

В рассматриваемой методике в качестве универсальных критериев для сравнительной оценки эффективности работы эксплуатационных подразделений различных перегонов и станций предлагаются следующие критерии, представляющие собой функции от Тв и То: относительный запас времени до восстановления работоспособности $\delta T_{B}^{\phi}$ и относительный запас среднего времени наработки на отказ $\delta T_{o}^{\phi}$

Отрицательные значения величин $\delta T_{o}^{\phi}$ и $\delta T_{B}^{\phi}$ сигнализируют о серьезных проблемах в реализации процесса технического обслуживания и необходимости пересмотра комплекса мероприятий по уменьшению времени устранения отказов СОДП на участке. В остальных случаях фактическое качество функционирования СОДП и уровень технологии технического обслуживания и ремонта на участке достаточны. Причем, чем выше значения $\delta T_{o}^{\phi}$ и $\delta T_{B}^{\phi}$, тем работа устройства надежнее, а технология обслуживания эффективнее.

Проанализируем в полной ли мере выбранные авторами методики показатели надежности могут оценить эффективность работы структурных подразделений по обслуживанию устройств инфраструктуры.

Как известно среднее время наработки на отказ определяется по формуле:

$$
T_{c p}=\frac{\sum_{i=1}^{N_{0}} t_{p_{i}}}{\sum_{i=1}^{N_{0}} n_{i}}
$$

Где $t_{p_{i}}$ - суммарное время безотказной работы $i$-го образца за время испытания;

$\sum_{i=1}^{N_{0}} t_{p_{i}}$ - уммарное время безотказной работы всех $N_{0}$ образов, участвующих в испытаниях, за время испытания;

$n_{i}$ - суммарное число отказов -го образца за время испытания. 
$\sum_{i=1}^{N_{0}} n_{i}$ - суммарное число отказов всех $N_{0}$ образцов, участвующих в испытаниях, за время испытания.

В случае если поток отказов является простейшим, то $T_{0}$ и $T_{c p}$ равны, то $K_{n}$ принято определять по формуле:

$$
K_{n}=\frac{T_{B}}{T_{o}+T_{B}}
$$

В соответствии с примером расчета [3] фактическое среднее время наработки на отказ определяется с учетом суммарной длительности отказов за расчетный период $-T$ :

Где $t$ - расчетный период,

$$
T_{o}=\frac{t-T}{N_{\text {omк }}}
$$

которая в свою очередь, отнесенная к количеству отказов за расчетный период $N_{\text {отк }}$, является фактическим средним временем восстановления для данного ТОИ:

$$
T_{B}=\frac{T}{N_{\text {om } \kappa}}
$$

Таким образом, среднее время наработки на отказ $T_{o}$, в соответствии с рассматриваемым примером расчета, является по сути функцией среднего времени восстановления $T_{\boldsymbol{B}}$.

В связи с этим представляется спорным использование среднего времени наработки на отказ $T_{o}$, как самостоятельного критерия для оценки эффективности организации процесса обслуживания технических средств.

На наш взгляд, показатель - среднее время восстановления $T_{6}$, может являться ключевым и единственным при оценки эффективности работы структурных подразделений по обслуживанию устройств инфраструктуры

Рассмотрим структуру данного показателя.

Как известно среднее время до восстановления работоспособности $T_{6}$ может быть разбито на следующие составляющие:

$$
T_{6}=t_{\phi}+t_{\text {изв }}+t_{c л}+t_{n}+t_{y}
$$

Где $t_{\phi}$-время фиксации (обнаружения) отказа;

$t_{\text {изв }}$ - время извещения обслуживающего персонала о возникновении отказа;

$t_{c л}$ - время следования обслуживающего персонала к месту возникновения отказа;

$t_{n}$ - время поиска причины отказа;

$t_{y}$ - время устранения отказа.

Из перечисленных составляющих, только две (время поиска причины отказа $t_{n}$, время устранения отказа $t_{y}$ ) напрямую связаны с квалификацией обслуживающего персонала. Следовательно, только они могут служить резервом времени, за счет которого без привлечения дополнительных ресурсов и возможностей (а только за счет повышения квалификации обслуживающего персонала) может быть сокращено фактическое среднее время до восстановления работоспособности $T_{B}^{\Phi}$. Оставшиеся составляющие (время фиксации (обнаружения) отказа $t_{\phi}$; время извещения

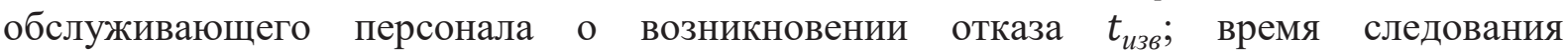
обслуживающего персонала к месту отказа $t_{c л}$ ) с квалификацией обслуживающего персонала не связаны. Данные составляющие определяются технической оснащенностью конкретного структурного подразделения, типом применяемых СОДП на станции или участке (наличием автоматизированных систем диспетчерского контроля, фактической системой организации технического обслуживания, наличием служебного автотранспорта и т.д.). Данные факторы, на наш взгляд, не исключаются введением величины относительного запаса времени до восстановления $\delta T_{b}^{\phi}$, и, следовательно, делают несоизмеримыми усилия различных структурных 
подразделений по повышению уровня технологии технического обслуживания и ремонта СОДП на участке или станции.

C учетом вышеизложенного, можно сделать вывод о необходимости корректировки предложенной методики по оценке эффективности работы структурных подразделений по обслуживанию устройств инфраструктуры.

$$
* * *
$$

1. Гапанович В.А., Безродный Б.Ф., Горелик А.В., Шалягин Д.В., Методология анализа работы структурных подразделений // Автоматика, связь, информатика.2013, №1, с. 2-5.

2. $\quad$ Гапанович В.А., Безродный Б.Ф., Горелик А.В., Шалягин Д.В. Внедрение методологии УРРАН в хозяйстве АТ // Автоматика, связь, информатика. 2012, № 4, с.12-15.

3. Методическое руководство по управлению ресурсами и рисками в хозяйстве автоматики и телемеханики на основе методологии УРРАН / ОАО «РЖД», Москва 2012, 167 с.

\section{Литвина М.И., Узликова П.Д. \\ Исследование системы мотивации на основе реализации социальной политики компании}

ФГБОУ ВО Московский автомобильно-дорожный государственный технический университет (МАДИ)

(Россия, Москва)

doi: 10.18411/lj-04-2021-279

\section{Аннотация}

Статья посвящена вопросам социальной ответственности бизнеса и важности социальной политики организации. Отмечена недостаточная степень изученности социальной политики организаций малого и среднего бизнеса. С помощью метода анкетного опроса авторы исследуют систему мотивации организации среднего бизнеса. На основе анализа результатов опроса предложены рекомендации по совершенствованию социальной политики организации. Представленная методика исследования может быть использована организациями различных сфер деятельности.

Ключевые слова: социальная политика, социальная ответственность, персонал, мотивация, мотивы труда, анкетный опрос, малый и средний бизнес

\section{Abstract}

The article is devoted to the issues of social responsibility of business and the importance of social policy of the organization. An insufficient degree of study of the social policy of small and medium-sized businesses is noted. Using the method of a questionnaire survey, the authors investigate the system of motivation for a medium-sized business organization. Based on the analysis of the survey results, recommendations for improving the social policy of the organization are proposed. The presented research methodology can be used by organizations in various fields of activity.

Keywords: social policy, social responsibility, personnel, motivation, motives of labor, questionnaire survey, small and medium-sized businesses

Одной из исторических причин возникновения социальной работы в организации в первой половине XIX века является идея улучшения условий труда работников.

В начале XX века значительных успехов добился Генри Форд. Он проводил эффективную социальную политику. Генри Форд стал первым бизнесменом, который внедрил особый вид внутрикорпоративной социальной ответственности перед работниками своих предприятий [2, с.18]. 